\title{
Clinical characteristics and prediction model of long time survival of patients with stage M1 Siewert type II esophagogastric junction adenocarcinoma
}

\author{
Jun Zhang, Deruo Liu \\ Department of Thoracic Surgery, Peking University China-Japan Friendship School of Clinical Medicine, Beijing, China \\ Contributions: (I) Conception and design: J Zhang; (II) Administrative support: D Liu; (III) Provision of study materials or patients: All authors; (IV) \\ Collection and assembly of data: J Zhang; (V) Data analysis and interpretation: J Zhang; (VI) Manuscript writing: All authors; (VII) Final approval of \\ manuscript: All authors. \\ Correspondence to: Deruo Liu. Department of Thoracic Surgery, Peking University China-Japan Friendship School of Clinical Medicine, No. 2, East \\ Yinghua Street, Chaoyang District, Beijing 100029, China. Email: deruoliu@163.com.
}

\begin{abstract}
Background: We aimed to study the clinical manifestations of the patients with stage M1 Siewert type II esophagogastric junction adenocarcinoma and more than 1-year overall survival and establish a prognosis prediction model
\end{abstract}

Methods: From the SEER database, 638 patients were chosen between 2011 and 2017. Patients were separated into two groups, including the long-time survival group ( $\geq 1$ year), and the shorter time survival group (<1 year). The analysis of differences in clinical characteristics (age, race, sex, stage T, stage N, grade, surgery, distant metastasis, survival status and time) between the different groups was performed by using the chi-square test. The predictors of overall survival was selected by using the Cox regression. The calibration curves and C-index were used to verify the nomogram.

Results: The chi-square test showed that the proportion of patients with the age of $\geq 65$ years in the long time survival group was lower than the short time survival group $(\mathrm{P}=0.008)$. The proportion of patients who received surgery was higher in the long time survival group $(13.5 \%$ vs. $5.3 \%, \mathrm{P}<0.001)$. There was a significantly lower proportions of bone metastasis in the long time survival group $(\mathrm{P}=0.036)$. Multivariate analyses indicated factors such as age, surgery, bone, liver, and lung metastasis were associated with prognosis. The C-index of the nomogram was 0.860 .

Conclusions: Age, surgery, bone, liver, and lung metastasis were related to the overall survival of a patient with stage M1 Siewert type II esophagogastric junction adenocarcinoma. We constructed a nomogram to help predict 1-year overall survival for a patient with stage M1 Siewert type II esophagogastric junction adenocarcinoma.

Keywords: Siewert type II; overall survival; SEER; esophagogastric junction adenocarcinoma; nomogram

Submitted Nov 19, 2020. Accepted for publication Apr 02, 2021.

doi: $10.21037 /$ tcr-20-3291

View this article at: http://dx.doi.org/10.21037/tcr-20-3291

\section{Introduction}

The incidence of adenocarcinomas of the esophagogastric junction are increasing in the whole wide world over the past two decades with a very poor 5-year overall survival for advanced esophagogastric junction adenocarcinoma (1-4). Siewert divided esophagogastric junction adenocarcinoma into three subgroups: Siewert types I (the distal esophagus adenocarcinoma with the epicenter situated within $1-5 \mathrm{~cm}$ above the esophagogastric junction), II (adenocarcinoma of the cardia with cancer epicenter located between 1-2 $\mathrm{cm}$ below the esophagogastric junction), and III (adenocarcinoma with cancer epicenter located between 
$2-5 \mathrm{~cm}$ below the esophagogastric junction) (5). About $30 \%$ of the patients were diagnosed with esophagogastric junction adenocarcinoma at an advanced stage with a rather poor prognosis. Moreover, many patients were presenting with metastases at the time of diagnosis with less than $11 \%$ of the overall 5 -year survival rate for the Siewert type II esophagogastric junction adenocarcinoma $(6,7)$. Given the special anatomic location, the prognosis of Siewert type II esophagogastric junction adenocarcinoma could be distinct from that of others (8-11). Although all cases are in stage M1, the survival time of patients varies. Some people lived for a long time, others lived for a short time. Some studies have shown that the prognosis of patients is related to their clinical characteristics. The study of Zhu K showed that the patients with Siewert type II esophagogastric junction adenocarcinoma had a favorable prognosis in the young group (9). The study of Chen K showed that patients who had bone metastases possessed worse cancer-specific survival than other distant metastasis. The purpose of the present study was to assess the clinical characteristics of the long-time survivors and establish a clinical nomogram by using the SEER database.

We present the following article in accordance with the TRIPOD reporting checklist (available at http://dx.doi. org/10.21037/tcr-20-3291).

\section{Methods}

\section{Research object}

For our study, the inclusion criteria were that (I) patients with M1 stage Siewert type II adenocarcinoma of the esophagogastric junction were diagnosed between 2010 and 2017; (II) the patients was older than 18; (III) patient information included age, race, sex, grade, stage $T$, stage $\mathrm{N}$, surgery, distant metastasis, survival status, survival time. Patients with incomplete information were excluded. The vast majority of patients with M1 stage Siewert type II adenocarcinoma of the esophagogastric junction live less than a year. Patients were separated into two groups, including the long-time survival group, and the shorter time survival group. Patients who survived for at least 1 year were assigned to the long-term survival group and those who survived for less than 1 year were assigned to the short-term survival group. The study was conducted in accordance with the Declaration of Helsinki (as revised in 2013). This article does not contain any studies with human participants performed by any of the authors. All procedures performed in study involving human data were extracted freely from the SEER Research Data available to the public online (https://seer.cancer.gov/data/access.html).

\section{Statistical analysis}

The analysis of differences in clinical characteristics between the different groups was performed by using the chi-square test. We evaluated the risk factors associated with overall survival using the Cox regression and $\mathrm{R}$ software was used to build a nomogram. $\mathrm{P}<0.05$ was regard as statistically significant. The results were assessed by using the calibration curve. The calibration of the nomogram was checked by using the calibration curves. By using the $\mathrm{R}$ software data analyses were all implemented.

\section{Results}

\section{Demographics of the patients}

Overall, 638 patients in the SEER database were extracted for analysis among which 185 (28.7\%) patients were in the long time survival group and $453(71.3 \%)$ were in a short time survival group. There was no statistical difference in sex, race, grade, $\mathrm{N}$ and $\mathrm{T}$ stage, brain, liver, and lung metastasis was found between the two groups. The proportion of patients with the age of $\geq 65$ years in a long time survival group was lower, compared to the short time survival group $(\mathrm{P}=0.008)$. Surgical information from the SEER database showed that patients in the long time survival group had a higher ratio of surgery (13.5\%, $5.3 \%, \mathrm{P}<0.001)$. There was some difference between the two groups in the distribution of distant metastasis. The proportion of patients with bone, brain, liver, and lung metastasis in the long time survival group was $16(8.6 \%)$, 4 (2.2\%), 99 (53.5\%), and 31 (16.8\%) respectively; while in the short time survivals group, the proportion was $67(14.8 \%), 14(3.1 \%), 252(55.6 \%)$, and $75(16.6 \%)$ respectively. The bone metastatic rate was lower in the long time survival group with statistical difference $(\mathrm{P}=0.036)$ (Table 1).

\section{Cox regression to analysis the risk factors}

The role of each variable in predicting overall survival was evaluated by using the Cox proportional hazards model. Multivariate analyses showed that age, surgery, bone, liver, and lung metastasis were related with prognosis of patients. 
Table 1 Demographic and clinicopathologic characteristics of the different overall survival

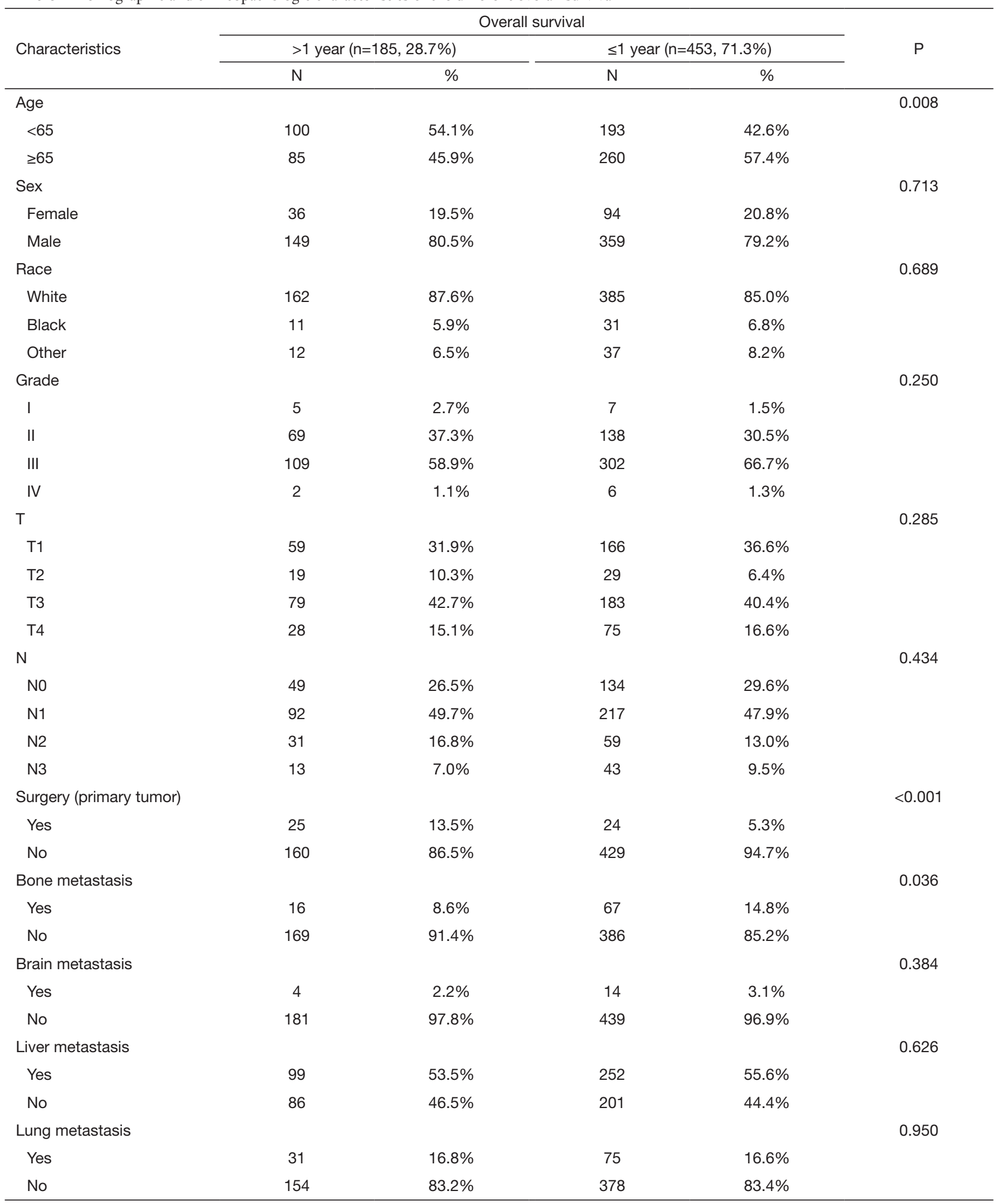




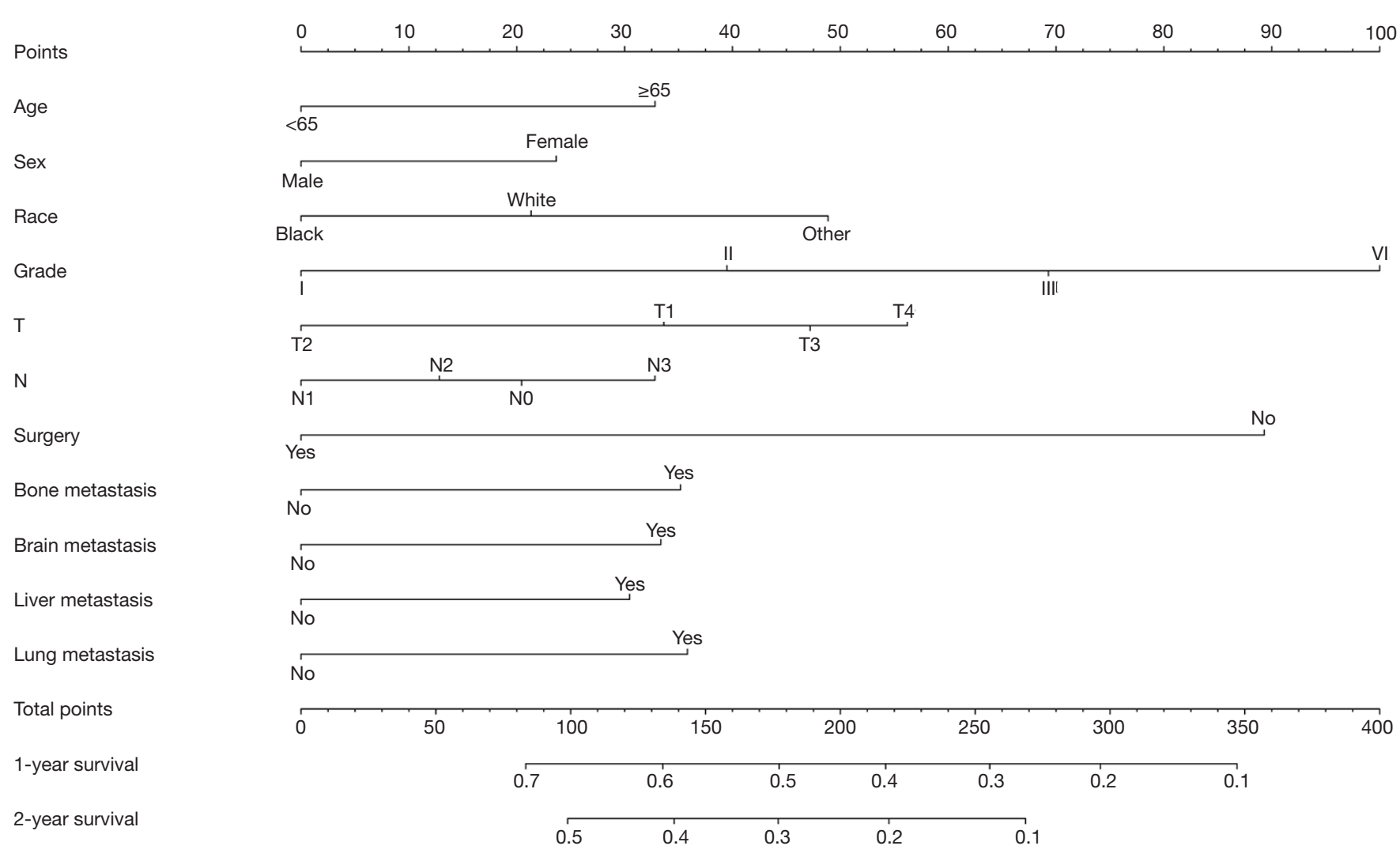

Figure 1 The nomogram that we built to predict the overall survival.

\section{The construction of novel nomogram}

The model that incorporated the above factors was developed and presented as the nomogram (Figure 1). Every predictor was scored according to a point scale. By using the total scores projected in the scale at the bottom, we could then foretell the probability of overall survival for patients with M1 stage Siewert type II adenocarcinoma of the esophagogastric junction. The C-index was 0.860 . The probability of 1- and 2-year overall survival of the calibration curves showed a good consistency (Figures 2,3).

\section{Discussion}

We investigated the difference between a long time survival group and a short time survival group and we also constructed a nomogram to help predict 1- and 2-year overall survival in patients with stage M1 Siewert type II esophagogastric junction adenocarcinoma. The results could assist doctor to choose the appropriate treatment strategies.

The function of the body's organs will decrease when people age with their unique characteristics of pathology, physiology, and metabolism. The study of Zhu K showed that the patients with Siewert type II esophagogastric junction adenocarcinoma had a favorable prognosis in the young group $(<65)$ than the old group $(\geq 65)$ with statistically significant (9). The research of Zhang et al. also demonstrated that patients with esophagogastric junction adenocarcinoma who were older than 65 years old had a worse prognosis than those under 65 years old, which was consistent with our study (12). The possible reasons were as follows. First of all, it might be related to the characteristics of the elderly patients themselves. Second, there was less possibility that older patients could receive surgery than young.

In our study, the most common site of metastasis was the liver, followed by lung, bone, and brain for the patients with stage M1 Siewert type II esophagogastric junction adenocarcinoma, which was similar to the reported findings $(13,14)$. The long time survival group had significantly lower proportions of bone metastasis than the short time survival group $(\mathrm{P}=0.036)$. The study of Chen $\mathrm{K}$ showed 


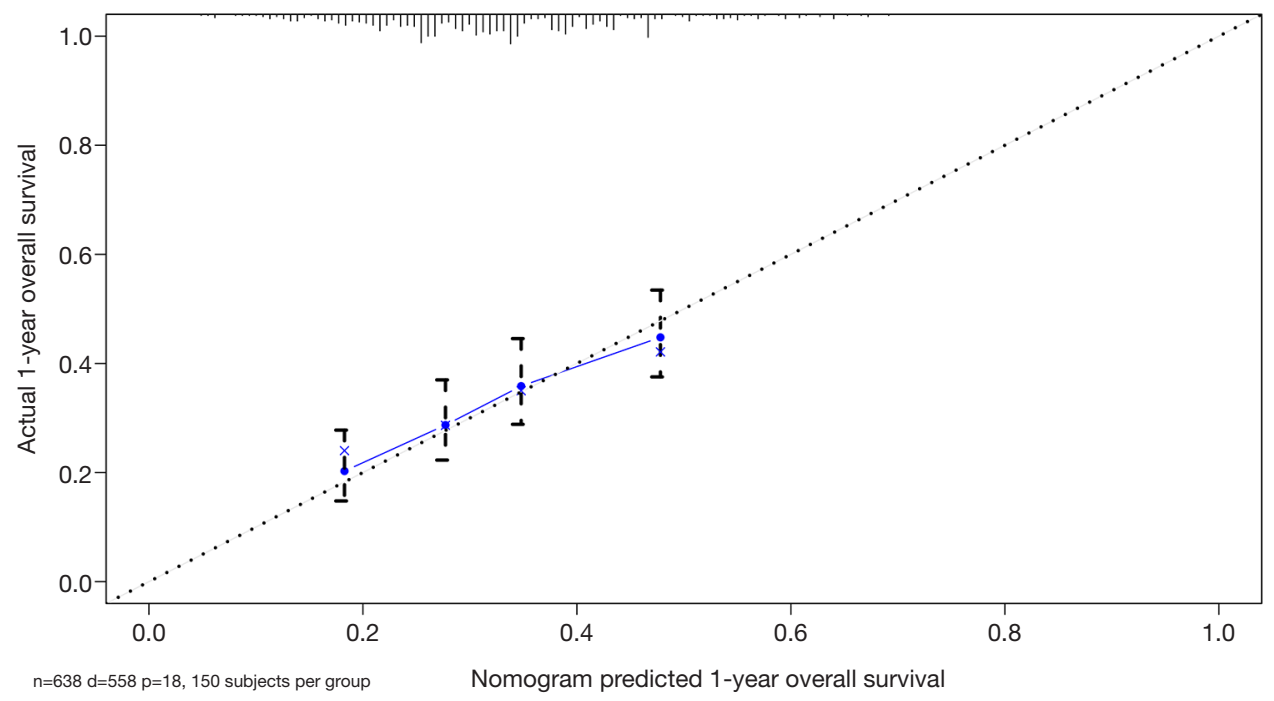

Figure 2 The calibration curve of nomogram-predicted 1-year overall survival.

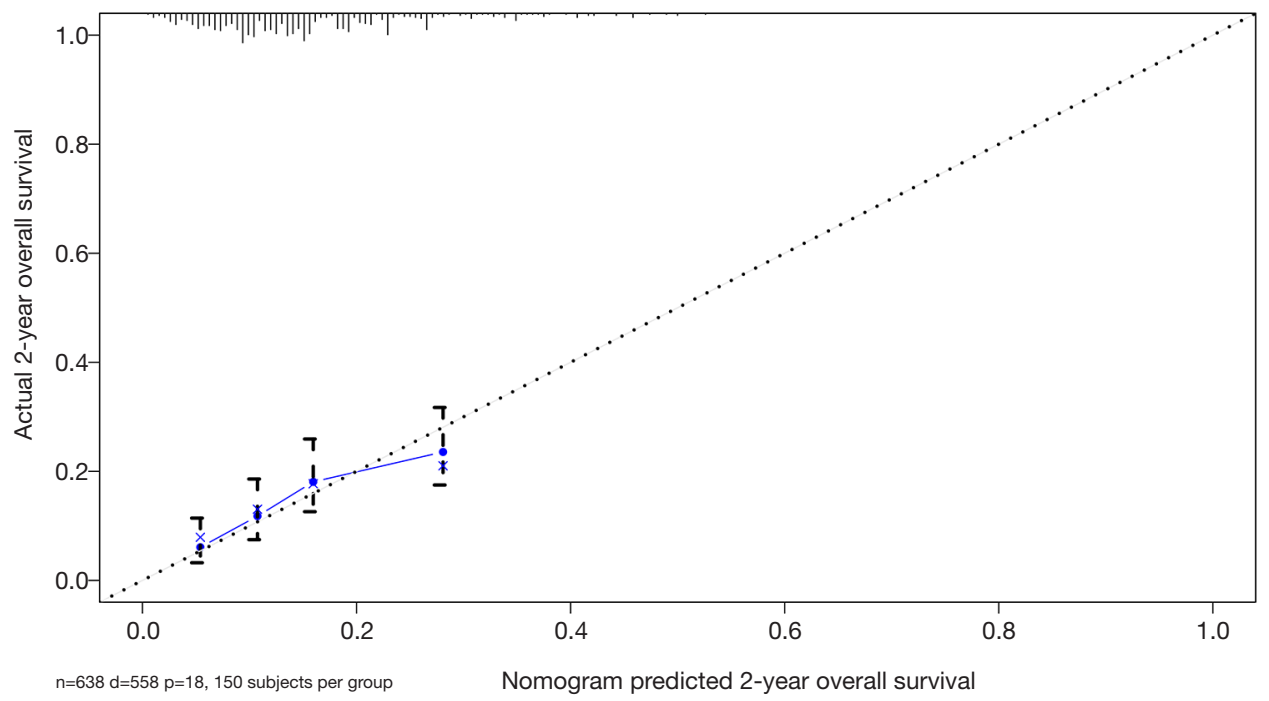

Figure 3 The calibration curve of nomogram-predicted 2-year overall survival.

that patients who had bone metastases possessed worse cancer-specific survival than other distant metastasis (15). Patients with bone metastasis from esophageal cancer had a poor prognosis (16). The reason that why bone metastasis could cause poor survival is indistinct. The study of Carmona-Bayonas et al. also demonstrated that the number of distant metastases was connected with overall survival (17). However, a previous report showed that the number of distant metastases and the metastatic site was not linked with overall survival (18). Similarly, the research of Blank et al. demonstrated that esophagogastric junction adenocarcinoma patients with distant metastases have poor prognoses, which had nothing to do with the metastatic site or the number of metastases (19). The reasons that could cause the inconsistency between the studies were might that the sample size was too small in the previous reported studies to ascertain the links.

Surgical information from the SEER database showed that patients in the long time survival group had a higher ratio of surgery $(13.5 \%, 5.3 \%, \mathrm{P}<0.001)$, which might mean 
that palliative surgical resection could improve survival in patients with stage M1 Siewert type II esophagogastric junction adenocarcinoma. The study of CarmonaBayonas et al. showed that in the patients with metastatic esophagogastric junction adenocarcinoma surgical resection might improve the prognosis of patients (14). Similarly, the study of the Riihimäki $M$ revealed that patients who had the localized metastatic cancers showed a satisfying survival when they received neoadjuvant chemotherapy followed by surgery (20). In the future, it still needed a large number of prospective randomized studies to prove that whether surgical resection would turn into an essential treatment in the patients with stage M1 Siewert type II esophagogastric junction adenocarcinoma.

For the study, there were still some limitations. First, because the study was a retrospective study, it will have an unavoidable confounding bias. In the second place, The population studied was mainly American and not representative of the global population. Finally, before the proposed nomogram could be used in clinical practice, it still needed to be replicated and verified prospectively.

\section{Conclusions}

In conclusion, age, surgery, bone, liver, and lung metastasis were related to the overall survival of a patient with stage M1 Siewert type II esophagogastric junction adenocarcinoma. We constructed a nomogram to predict 1-year overall survival for a patient with stage M1 Siewert type II esophagogastric junction adenocarcinoma. Clinicians can use the prognostic model to predict a patient's prognosis.

\section{Acknowledgments}

We thank Steven Wong for providing language editing assistance in this study.

Funding: None.

\section{Footnote}

Reporting Checklist: The authors have completed the TRIPOD reporting checklist. Available at http://dx.doi. org/10.21037/tcr-20-3291

Conflicts of Interest: Both authors have completed the ICMJE uniform disclosure form (available at http://dx.doi. org/10.21037/tcr-20-3291). The authors have no conflicts of interest to declare.

Ethical Statement: The authors are accountable for all aspects of the work in ensuring that questions related to the accuracy or integrity of any part of the work are appropriately investigated and resolved. The study was conducted in accordance with the Declaration of Helsinki (as revised in 2013). This article does not contain any studies with human participants performed by any of the authors. All procedures performed in study involving human data were extracted freely from the SEER Research Data available to the public online (https://seer.cancer.gov/data/ access.html).

Open Access Statement: This is an Open Access article distributed in accordance with the Creative Commons Attribution-NonCommercial-NoDerivs 4.0 International License (CC BY-NC-ND 4.0), which permits the noncommercial replication and distribution of the article with the strict proviso that no changes or edits are made and the original work is properly cited (including links to both the formal publication through the relevant DOI and the license). See: https://creativecommons.org/licenses/by-nc-nd/4.0/.

\section{References}

1. Amini N, Spolverato G, Kim Y, et al. Clinicopathological features and prognosis of gastric cardia adenocarcinoma: a multi-institutional US study. J Surg Oncol 2015;111:285-92.

2. Matsuno K, Ishihara R, Ohmori M, et al. Time trends in the incidence of esophageal adenocarcinoma, gastric adenocarcinoma, and superficial esophagogastric junction adenocarcinoma. J Gastroenterol 2019;54:784-91.

3. Karstens KF, Ghadban T, Sawez S, et al. Comparison of the 8th UICC staging system for esophageal and gastric cancers in Siewert type II junctional adenocarcinomas. Eur J Surg Oncol 2020;46:638-43.

4. Hasegawa S, Yoshikawa T. Adenocarcinoma of the esophagogastric junction: incidence, characteristics, and treatment strategies. Gastric Cancer 2010;13:63-73.

5. Rüdiger Siewert J, Feith M, Werner M, et al. Adenocarcinoma of the esophagogastric junction: results of surgical therapy based on anatomical/topographic classification in 1,002 consecutive patients. Ann Surg 2000;232:353-61.

6. Jim MA, Pinheiro PS, Carreira H, et al. Stomach cancer survival in the United States by race and stage (2001-2009): Findings from the CONCORD-2 study. Cancer 2017;123 
Suppl 24:4994-5013.

7. Suzuki A, Xiao L, Hayashi Y, et al. Nomograms for prognostication of outcome in patients with esophageal and gastroesophageal carcinoma undergoing definitive chemoradiotherapy. Oncology 2012;82:108-13.

8. Curtis NJ, Noble F, Bailey IS, et al. The relevance of the Siewert classification in the era of multimodal therapy for adenocarcinoma of the gastro-oesophageal junction. J Surg Oncol 2014;109:202-7.

9. Zhu K, Xu Y, Fu J, et al. Proximal Gastrectomy versus Total Gastrectomy for Siewert Type II Adenocarcinoma of the Esophagogastric Junction: A Comprehensive Analysis of Data from the SEER Registry. Dis Markers 2019;2019:9637972.

10. Hasegawa S, Yoshikawa T, Cho H, et al. Is adenocarcinoma of the esophagogastric junction different between Japan and western countries? The incidence and clinicopathological features at a Japanese high-volume cancer center. World J Surg 2009;33:95-103.

11. Hasegawa S, Yoshikawa T, Aoyama T, et al. Esophagus or stomach? The seventh TNM classification for Siewert type II/III junctional adenocarcinoma. Ann Surg Oncol 2013;20:773-9.

12. Zhang H, Wang W, Cheng Y, et al. Adenocarcinomas of the esophagogastric junction: experiences at a single institution in China. World J Surg Oncol 2013;11:155.

13. Chen K, Deng X, Yang Z, et al. Survival nomogram for patients with metastatic Siewert type II adenocarcinoma of the esophagogastric junction: a population-based study.
Expert Rev Gastroenterol Hepatol 2020;14:757-64.

14. Chen K, Deng X, Yang Z, et al. Sites of distant metastases and the cancer-specific survival of metastatic Siewert type II esophagogastric junction adenocarcinoma: a population-based study. Expert Rev Gastroenterol Hepatol 2020;14:491-7.

15. Liu F, Zhou R, Jiang F, et al. Proposal of a Nomogram for Predicting Survival in Patients with Siewert Type II Adenocarcinoma of the Esophagogastric Junction After Preoperative Radiation. Ann Surg Oncol 2019;26:1292-300.

16. Dhupar R, Rice RD, Correa AM, et al. Endoscopic Ultrasound Estimates for Tumor Depth at the Gastroesophageal Junction Are Inaccurate: Implications for the Liberal Use of Endoscopic Resection. Ann Thorac Surg 2015;100:1812-6.

17. Carmona-Bayonas A, Jiménez-Fonseca P, Echavarria I, et al. Surgery for metastases for esophageal-gastric cancer in the real world: Data from the AGAMENON national registry. Eur J Surg Oncol 2018;44:1191-8.

18. Fornaro L, Fanotto V, Musettini G, et al. Selecting patients for gastrectomy in metastatic esophago-gastric cancer: clinics and pathology are not enough. Future Oncol 2017;13:2265-75.

19. Blank S, Lordick F, Dobritz M, et al. A reliable risk score for stage IV esophagogastric cancer. Eur J Surg Oncol 2013;39:823-30.

20. Riihimäki $M$, Hemminki A, Fallah $M$, et al. Metastatic sites and survival in lung cancer. Lung Cancer 2014;86:78-84.
Cite this article as: Zhang J, Liu D. Clinical characteristics and prediction model of long time survival of patients with stage M1 Siewert type II esophagogastric junction adenocarcinoma. Transl Cancer Res 2021;10(5):2002-2008. doi: 10.21037/tcr-203291 